\title{
BALANCED COLOURINGS AND THE FOUR COLOUR CONJECTURE ${ }^{1}$
}

\author{
J. A. BONDY
}

ABSTRACT. A conjectured property of bridgeless cubic planar graphs is shown to be equivalent to the four colour conjecture. In establishing this equivalence use is made of the König-Hall theorem on the existence of one-factors in bipartite graphs.

1. The four colour conjecture. For a discussion of the four colour conjecture and related topics we refer the reader to Ore [3]. We merely content ourselves here with a statement of the conjecture and of two equivalent conjectures (proofs of their equivalence are given in [3]). Our graph-theoretic terminology is that of Harary [2] (although we use vertices and edges for what are respectively called points and lines in [2]).

FOUR COLOUR CONJEC.TURE. The faces of any plane graph can be coloured with four colours so that no two incident faces are assigned the same colour.

The HeAwOOd CONJECTURE. For any bridgeless cubic plane graph $G$ there is a mapping $f: V(G) \rightarrow\{-1,+1\}$ so that, for every face $F, \sum_{x \in F} f(x) \equiv$ $0(\bmod 3)$.

The TAIT CONJECTURE. Every bridgeless cubic planar graph has an even two-factor (that is a two-factor in which each cycle is of even length).

2. Balanced colourings. Let $G$ be a graph with vertex set $V(G)$ and edge set $E(G)$. A partition $\mathscr{C}=(B, W)$ of $V(G)$ is a two-colouring of $V(G) ; \mathscr{C}$ is an equitable two-colouring if $|B|=|W|$. For a two-colouring $\mathscr{C}$ and a vertex $x$, we define $w(x, \mathscr{C})$, the weight of $x$ in $\mathscr{C}$, by

$$
\begin{aligned}
w(x, \mathscr{C})=-2 & \text { if } x \in B, \\
=+2 & \text { if } x \in W,
\end{aligned}
$$

and for $S \subseteq V(G)$ we denote $\sum_{x \in S} w(x, \mathscr{C})$ by $w(S, \mathscr{C})$.

Again, for $S \subseteq V(G)$, let $v(S)$ denote the number of edges of $G$ having exactly one end in $S$. A balanced colouring of $G$ is a two-colouring $\mathscr{C}$ of

Received by the editors August 2, 1971.

AMS 1970 subject classifications. Primary 05C15.

Key words and phrases. Balanced colouring, four colour conjecture, bridgeless cubic planar graphs, further, one-factor, even two-factor.

1 This paper was written while the author was a member of the 1971 C.M.C. Summer Research Institute at Université Laval.

(c) American Mathematical Society 1972 
$V(G)$ such that

$$
v(S) \geqq|w(S, \mathscr{C})| \quad \text { for all } S \subseteq V(G) .
$$

Two remarks about balanced colourings will serve to indicate why they are so named.

REMARK 1. If $\mathscr{C}$ is a balanced colouring, then $\mathscr{C}$ is equitable. (This is seen by taking $S=V(G)$ in $\left({ }^{*}\right)$.)

REMARK 2. In a balanced colouring of a cubic graph there can be no monochromatic (all $B$ or all $W$ ) path of three vertices.

We now state the

BALANCED COLOURING CONJECTURE. Every bridgeless cubic planar graph has a balanced colouring. (It should be noted that the two-colouring of the Heawood conjecture is not in general a balanced colouring.)

THEOREM. The balanced colouring conjecture is equivalent to the four colour conjecture.

Before giving the proof of this theorem we first note some simple facts concerning graphs having balanced colourings. Their proofs are omitted.

LEMMA 1. A cycle of even length has a balanced colouring (the vertices coloured alternately $B$ and $W$ ).

LEMMA 2. If every component of a graph $G$ has a balanced colouring, then so does $G$.

LEMMA 3. If a partial graph $G^{\prime}$ of $G$ has a balanced colouring, then so does $G$.

We shall also need the

KöNig-HALl TheOREM (see [1, p. 128]). A bipartite graph with parts $X$ and $Y$ has $a$ one-factor if and only if $|X|=|Y|$ and $|N(S)| \geqq|S|$ for all $S \subseteq X$, where $N(S)$ is the set of vertices adjacent to vertices of $S$.

G

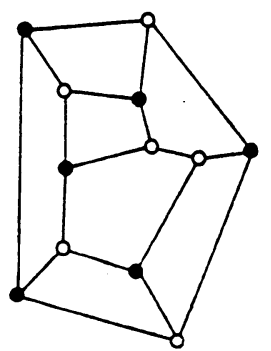

(a)
$G^{\prime}$

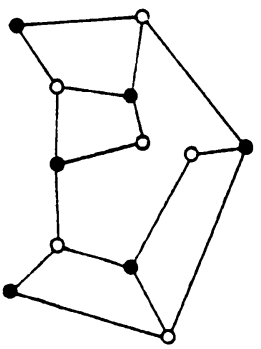

(b)
$G^{\prime \prime}$

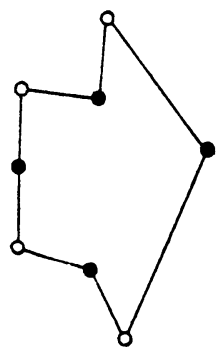

(c)

FIGURE 1 
PROOF of THEOREM. We show that the balanced colouring conjecture is equivalent to the Tait conjecture. Let $G$ be a bridgeless cubic planar graph. Suppose first that the Tait conjecture is true. Then $G$ has an even twofactor. By Lemmas $1-3, G$ has a balanced colouring. Now suppose that the balanced colouring conjecture is true and let $\mathscr{C}=(B, W)$ be a balanced colouring of $G$. We must show that $G$ has an even two-factor. Let $G^{\prime}$ be the partial graph of $G$ in which $(x, y) \in E\left(G^{\prime}\right)$ if and only if the weight of $\{x, y\}$ in $\mathscr{C}$ is zero (Figure $1(\mathrm{~b})$ ). Clearly $G^{\prime}$ is bipartite with parts $B, W$. By Remark 2 , in $G^{\prime}$ each vertex has degree two or three. Let $G^{\prime \prime}$ be the subgraph of $G^{\prime}$ spanned by the vertices of degree three in $G^{\prime}$ (Figure 1(c)).

We shall show that $G^{\prime \prime}$ has a one-factor. For suppose otherwise. Then, by the König-Hall theorem there are subsets $P, Q$ of $V\left(G^{\prime \prime}\right)$, of different colours, such that $|P|>|Q|$ and such that the only vertices adjacent to vertices of $P$ in $G^{\prime \prime}$ are vertices of $Q$. We can assume without loss of generality that $P \subseteq B, Q \subseteq W$. Now let $R$ be the set of $W$-vertices not in $V\left(G^{\prime \prime}\right)$ (that is, the set of $W$-vertices of degree two in $G^{\prime}$ ). Then the set $S=$ $P \cup Q \cup R$ violates (*). For, if $|P|=p,|Q|=q,|R|=r, w(S, \mathscr{C})=2(q+r-p)$. But, since vertices of $P$ are joined in $G$ only to vertices of $Q \cup R, v(S) \leqq$ $3 q+2 r-3 p<2(q+r-p)$. Hence $G^{\prime \prime}$ has a one-factor.

Let $E^{\prime}$ be the set of edges of this one-factor. Then $G^{\prime}-E^{\prime}$ is a partial subgraph of $G$, regular of degree two and bipartite; it is thus an even two-factor of $G$.

3. The four colour theorem? It now remains to prove the balanced colouring conjecture. Since every bridgeless cubic planar graph has a twofactor [4], there is a natural way of equitably two-colouring the verticescolour the vertices of each cycle of the two-factor alternately $B$ and $W$ (with two adjacent $B$ 's or $W$ 's for cycles of odd length). Such a twocolouring will always be 'nearly' balanced.

QUESTION 1. Is there necessarily a balanced colouring of this type?

The concept of a balanced colouring also leads us to the following question.

QUESTION 2. Does every bridgeless cubic planar graph have an equitable two-colouring without monochromatic paths of three vertices?

By Remark 2 the truth of the four colour conjecture would supply an affirmative answer to this question.

An alternative approach might be to study which graphs in general have balanced colourings. From Remark 1 it is clear that only graphs of even order need be considered; also, by taking $S$ to be a singleton set in (*), it is easily seen that every vertex must have degree at least two. In fact, by Lemma 3, we can restrict our attention to the maximal graphs having no balanced colouring (or to the minimal graphs that have balanced colourings). The Petersen graph is one such graph. 


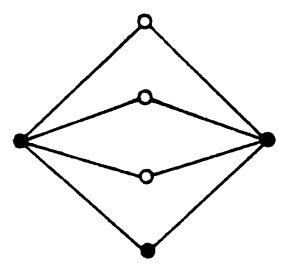

FIGURE 2

Lemmas 1-3 imply that every graph having an even two-factor possesses a balanced colouring. It is interesting to note that the converse is not true. For example $K_{2,4}$ has a balanced colouring but no two-factor (Figure 2).

I wish to thank Dr. Neil Robertson for kindly pointing out some simplifications to my original proof of the theorem.

\section{REFERENCES}

1. C. Berge, Graphes et hypergraphes, Dunod, Paris, 1971.

2. F. Harary, Graph theory, Addison-Wesley, Reading, Mass., 1969. MR 41 \#1566.

3. O. Ore, The four-color problem, Pure and Appl. Math., vol. 27, Academic Press, New York, 1967. MR 36 \#74.

4. J. Petersen, Die Theorie der regulären Graphen, Acta Math. 15 (1891), 193-220.

Faculty of Mathematics, University of Waterloo, Waterloo, Ontario, CANADA 